\title{
Counterion crossbridges enable robust multiscale elasticity in actin networks
}

\author{
Bekele Gurmessa, ${ }^{1}$ Madison Francis, ${ }^{1}$ Michael J. Rust, ${ }^{2}$ Moumita Das, ${ }^{3}$ Jennifer L. Ross $\odot,{ }^{4}$ \\ and Rae M. Robertson-Anderson (1),* \\ ${ }^{1}$ Department of Physics and Biophysics, University of San Diego, 5998 Alcala Park, San Diego, California 92110, USA \\ ${ }^{2}$ Department of Molecular Genetics and Cell Biology, University of Chicago, 900 East 57th Street, Chicago, Illinois 60637, USA \\ ${ }^{3}$ School of Physics and Astronomy, Rochester Institute of Technology, 84 Lomb Memorial Drive, Rochester, New York 14623, USA \\ ${ }^{4}$ Department of Physics, University of Massachusetts, Amherst, 666 North Pleasant Street, Amherst, Massachusetts 01003, USA
}

(Received 28 June 2019; published 28 August 2019)

\begin{abstract}
Bundling and crosslinking of actin networks provide cells with tensile strength and mobility and play critical roles in diverse mechanical processes such as wounding healing and fertilization. One simple mechanism for bundling and crosslinking actin networks is using divalent counterions such as $\mathrm{Mg}^{2+}$ to form crossbridges between actin filaments. Counterion crossbridges can also be exploited in materials engineering to alter the mechanics of polyelectrolyte materials. As such, the mechanical properties that counterion crossbridges confer to actin networks needs to be explored. Here, we use optical tweezers microrheology to characterize the mechanical response of actin networks in the presence of varying concentrations of $\mathrm{Mg}^{2+}$. We couple mechanics to structure by using confocal microscopy to characterize the mobility and architecture of networks. We show that only modest bundling and network rearrangement is required to induce dramatic increases in the elasticity and stiffness of the networks. We further show that bundles are resilient to nonlinear forcing and exhibit surprisingly minimal dissipation above a critical counterion concentration. Finally, we demonstrate that while crosslinking of network fibers plays an important role in linear regime mechanics, filament bundling dictates the nonlinear response.
\end{abstract}

DOI: 10.1103/PhysRevResearch.1.013016

\section{INTRODUCTION}

Semiflexible actin filaments are one of the most abundant biopolymers in the cytoskeleton, playing key roles in cell structure, adhesion, motility, and division [1]. Actin filaments are able to mediate these diverse mechanical processes and properties by forming entangled, crosslinked, and bundled networks with distinct mechanics and structures. For instance, networks of actin bundles comprise cellular structures such as stress fibers, filopodia, microvilli, and stereocilia; and enable mechanotransduction, fertilization, neuronal growth, and wound healing [2-4]. The unique bending stiffness, elasticity, and tensile strength of bundled networks confer the wideranging mechanical processes they enable [5,6]. While certain actin binding proteins (ABPs) can bundle actin filaments [4], due to the polyelectrolyte nature of actin, moderate concentrations of divalent ions, such as $\mathrm{Mg}^{2+}$, are also sufficient to bundle actin and form connected networks of bundles via counterion crossbridges [6-9]. More generally, counterion crossbridges offer a simple mechanism to tune the stiffness and connectivity of a broad class of entangled biopolymer and polyelectrolyte networks.

\footnotetext{
*randerson@ sandiego.edu
}

Published by the American Physical Society under the terms of the Creative Commons Attribution 4.0 International license. Further distribution of this work must maintain attribution to the author(s) and the published article's title, journal citation, and DOI.
Bundles of actin filaments are inherently stiffer than single filaments, such that a network of counterion-crossbridged filaments should have distinct mechanical properties from a network of single filaments [10]. For example, $\mathrm{Mg}^{2+}$ concentrations of $10-50 \mathrm{mM}$ have been reported to produce actin bundles with persistence lengths $l_{p}$ up to $\sim 4 \times$ larger than that of single actin filaments $\left(l_{p} \approx 10 \mu \mathrm{m}\right)[9,11-14]$. Further, theoretical studies have shown that the bending stiffness of a bundle is impacted by both the number of filaments comprising the bundle as well as the density of connections the bundle has with its neighbors, suggesting that both bundling as well as crosslinking of bundles may play an important role in the mechanics of counterion-crossbridged networks [5].

Due to the physiological importance of counterion crossbridging, as well as its importance in addressing open questions in polymer physics, several studies have explored the structural properties of actin bundles and networks formed in the presence of $\mathrm{Mg}^{2+}$ [2,6-9]. These studies have reported critical $\mathrm{Mg}^{2+}$ concentrations of $\sim 10-25 \mathrm{mM}$ for the onset of bundling, but the extent to which bundle size and connectivity increases with increasing salt concentration above this threshold remains debated $[2,9,15]$. Two studies have reported that bundles assume a finite size that is insensitive to the concentration of actin and divalent salt [16,17] while another study reported a nonmonotonic dependence of bundle size on salt concentration, reaching a peak at $30 \mathrm{mM}$ [9]. These findings are at odds with those from ABP-bundled actin network studies that reported a power-law increase in bundle size and stiffness with an increasing ABP:actin ratio 
$R[17,18]$. However, none of these studies have investigated how varying concentrations of divalent counterions impacts the mechanical properties of actin networks.

Nonetheless, several studies have probed the mechanical properties of actin networks bundled via different ABPs $[11,19,20]$. At sufficiently high $R$ values, scruin-crosslinked networks have been reported to exhibit stress-stiffening due to the stretching of bundles, whereas low $R$ networks displayed stress-softening arising from bending [21,22]. In contrast, actin networks bundled with fascin or varying mutants of $\alpha$-actinin [23] have been reported to stiffen at low $R$ and soften at high $R$ due to the rupturing of crosslinks that connected discrete bundles. These studies, as well as related simulation studies [24], have reported that the magnitudes of the frequency-dependent viscoelastic moduli $G^{\prime}(\omega)$ and $G^{\prime \prime}(\omega)$ increased with increasing crosslinking, with all networks exhibiting a similar scaling of $G^{\prime}$ and $G^{\prime \prime}$ with frequency that is unique from that of entangled networks. In the low-frequency regime, both $G^{\prime}$ and $G^{\prime \prime}$ scale as $\omega^{1 / 2}$. At intermediate frequencies, $G^{\prime}>G^{\prime \prime}$ with $G^{\prime}$ exhibiting an $\omega$-independent plateau while $G^{\prime \prime}$ reaches a local maximum followed by a minimum. In the high-frequency limit, $G^{\prime \prime}>G^{\prime}$ with both moduli exhibiting power-law scaling with exponents of $\sim 0.5-1$. While the high-frequency limit has been predicted to reflect the dynamics of single filaments or bundles, the low- $\omega$ regime displays the rheology of the connected network. The extent to which these mechanical properties translate to counterionconnected networks remains unknown. While the transient nature of $\mathrm{ABP}$ crosslinkers has been suggested to be primarily responsible for the mechanics described above, it is unclear if counterion crossbridges exhibit similar plasticity and nonequilibrium dynamics.

Here, we use optical tweezers microrheology and confocal microscopy to characterize the linear and nonlinear mechanical response as well as the mobility and architecture of entangled actin networks in the presence of varying concentrations of $\mathrm{Mg}^{2+}$. To probe the nonlinear force response, we optically drag a microsphere through the network and measure the resulting force the network exerts on the sphere during and after the strain [25]. To measure the linear response, we track the thermal fluctuations of trapped microspheres to quantify the frequency-dependent viscoelastic moduli $G^{\prime}(\omega)$ and $G^{\prime \prime}(\omega)$. We couple these measurements with laser scanning confocal microscopy experiments to characterize the effect of $\mathrm{Mg}^{2+}$ on the network microstructure and rigidity.

We show that modest increases in salt concentration (2-52 $\mathrm{mM}$ ) lead to dramatic changes in the mechanical response-with network stiffness, nonlinear force response, elasticity, and relaxation timescales all increasing by an order of magnitude or more. Unlike actin networks crosslinked by ABPs, counterion-bundled networks exhibit a nearly complete elastic response with essentially no dissipation. While such extreme behavior is typically expected to arise from dramatic changes to the network architecture, we instead find that the induced bundling and crosslinking results in relatively small changes to the mesoscopic structure of the network. We also show that while crosslinking of network fibers plays an important role in linear regime mechanics, filament bundling - which only occurs above a critical salt concentration-dictates the nonlinear response.

\section{MATERIALS AND METHODS}

\section{A. Sample preparation}

Alexa-568-labeled actin (A-12373, Life Technologies) and unlabeled actin monomers (Cytoskeleton, Inc.) were stored at $-80^{\circ} \mathrm{C}$ in G-buffer ( $2 \mathrm{mM}$ Tris $p \mathrm{H}$ 8.0, $0.5 \mathrm{mM}$ DTT, $0.1 \mathrm{mM}$ $\mathrm{CaCl}_{2}$ ). To form networks, thawed Alexa-568-actin and unlabeled G-actin were mixed at a 1:10 molar ratio to a final concentration of $5.8 \mu \mathrm{M}$ in $100 \mathrm{mM}$ K-Pipes $p \mathrm{H} 7.0,2 \mathrm{mM}$ EGTA, $1 \mathrm{mM}$ ATP, and varying concentrations of $\mathrm{MgCl}_{2}(2,4$, 14,27 , or $52 \mathrm{mM}$ ). A trace amount of Alexa 488-BSA-coated polystyrene microspheres (Polysciences, Inc.) of $4.5 \mu \mathrm{m}$ in diameter was added for microrheology measurements (Fig. 1). The solution was pipetted into a microscope sample chamber comprised of double-sided tape as a spacer between the slide and coverslip, sealed with epoxy, and incubated for $1 \mathrm{~h}$ at room temperature. The predicted mesh size of the network is $\xi=0.3 / c_{a}^{1 / 2}=0.6 \mu \mathrm{m}$, where $c_{a}$ is the actin concentration in $\mathrm{mg} / \mathrm{mL}$.

\section{B. Microrheology}

We used an optical trap setup built by outfitting an Olympus IX71 fluorescence microscope with a 1064-nm ND:YAG fiber laser (Manlight) focused with a $60 \times 1.4$ numerical aperture (NA) objective, as described and validated previously [25,26]. A position-sensing detector (First Sensor) measured the laser deflection, which is proportional to the force exerted on a trapped microsphere by the network. The trap stiffness was calibrated via Stokes drag in water and passive equipartition methods [27]. The laser deflection was recorded at a rate of $20 \mathrm{kHz}$ using custom-written LABVIEW programs. Custom MATLAB scripts were used for postacquisition data analysis. All presented data are averages of 20 trials using 20 different beads each at a different location in the sample chamber.

For linear microrheology measurements [Fig. 1(c)], $G^{\prime}(\omega)$ and $G^{\prime \prime}(\omega)$ were extracted from the thermal fluctuations of an optically trapped bead (recorded for $180 \mathrm{~s}$ ) using the generalized Stokes-Einstein relation as described in Ref. [28]. Briefly, the normalized mean-squared displacement of the ensemble of trapped beads $\Pi(\tau)=\left\langle\Delta r^{2}(\tau)\right\rangle / 2\left\langle r^{2}\right\rangle$ is computed, from which the Fourier transform of $\Pi(\tau), \hat{\Pi}(\omega)$, is computed via the relationship [28]

$$
\begin{gathered}
-\omega^{2} \hat{\Pi}(\omega) \frac{\Pi(\tau)}{\tau_{1}}\left(1-e^{-i \omega \tau_{1}}\right)+\dot{\Pi}_{\infty} e^{-i \omega \tau_{N}} \\
+\sum_{k=2}^{N}\left(\frac{\Pi_{k}-\Pi_{k-1}}{\tau_{k}-\tau_{k-1}}\right)\left(e^{-i \omega \tau_{k-1}}-e^{-i \omega \tau_{k}}\right),
\end{gathered}
$$

where $\dot{\Pi}_{\infty}$ is the slope of $\Pi(\tau)$ extrapolated to infinite time and $t_{N}$ is the $N$ th lag time. The complex modulus $G^{*}(\omega)=$ $G^{\prime}(\omega)+i G^{\prime \prime}(\omega)$ is then determined using $G^{*}(\omega) 6 \pi a / k=$ $[1 / \omega \hat{\Pi}(\omega)-1]$, where $a$ is the bead radius and $k$ is the trap stiffness.

For nonlinear microrheology [Fig. 1(d)], a piezoelectric nanopositioning stage (Mad City Laboratories) displaced an optically trapped bead $5 \mu \mathrm{m}$ relative to the sample chamber at a speed of $20 \mu \mathrm{m} / \mathrm{s}$ while measuring the resulting force the network exerted on the trapped bead during $(0.25 \mathrm{~s})$ and after 
(a)
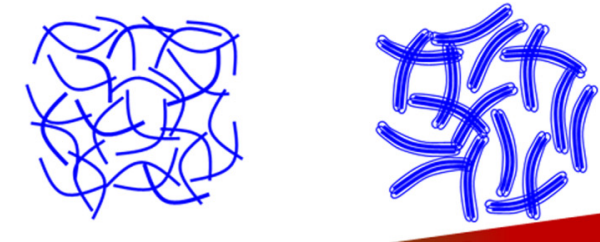

(b)

(c)
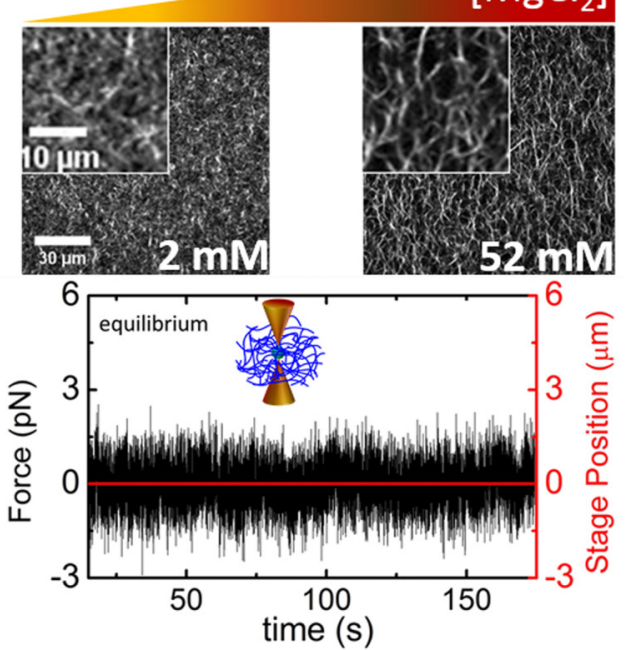

(d)

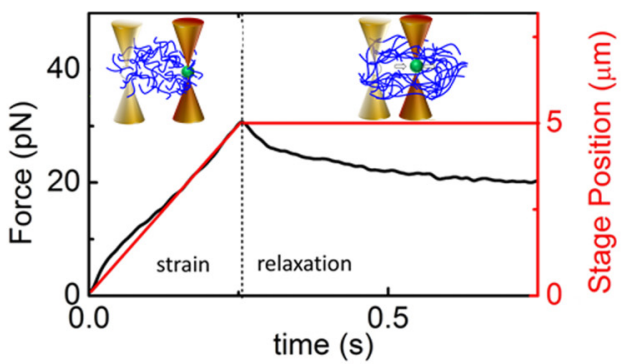

FIG. 1. Experimental approach to elucidate the effect of counterion crossbridges on the mechanics and structure of entangled actin networks. (a) Cartoon of networks of single actin filaments at low $\left[\mathrm{MgCl}_{2}\right]$ (left) and networks of bundles at high $\left[\mathrm{MgCl}_{2}\right]$ (right). (b) Projection of 900 frames of a representative time series of $5.8-\mu \mathrm{M}$ actin networks with $\left[\mathrm{MgCl}_{2}\right]$ listed. Time series were captured at 15 fps using a Nikon A1R confocal microscope and QImaging CCD camera. (c), (d) Optical tweezers microrheology measurements showing sample measured force traces (black) and stage position (red) for (c) linear microrheology determined from thermal fluctuations of a trapped bead, and (c) nonlinear microrheology in which the bead is displaced $5 \mu \mathrm{m}$ at $20 \mu \mathrm{m} / \mathrm{s}$ (strain) after which the force is recorded for an additional $30 \mathrm{~s}$ (relaxation). Data shown are for a network with $14 \mathrm{mM} \mathrm{MgCl}_{2}$.

(30 s) the strain. The speed was chosen to ensure we were accessing the nonlinear regime [25].

\section{Microscopy}

Time-series and three-dimensional image stacks ( $z$-stacks) of networks were collected using a Nikon A1R laser scanning confocal microscope with a $60 \times 1.4$ NA objective and QImaging CCD camera [Fig. 1(b)]. The $512 \times 512$ pixel time-series was recorded at $15 \mathrm{fps}$ for $60 \mathrm{~s}$. The $z$-stacks comprised 100 images with $0.2-\mu \mathrm{m}$ spacing. Using Fiji, the projection of the mean intensity of each pixel of each time-

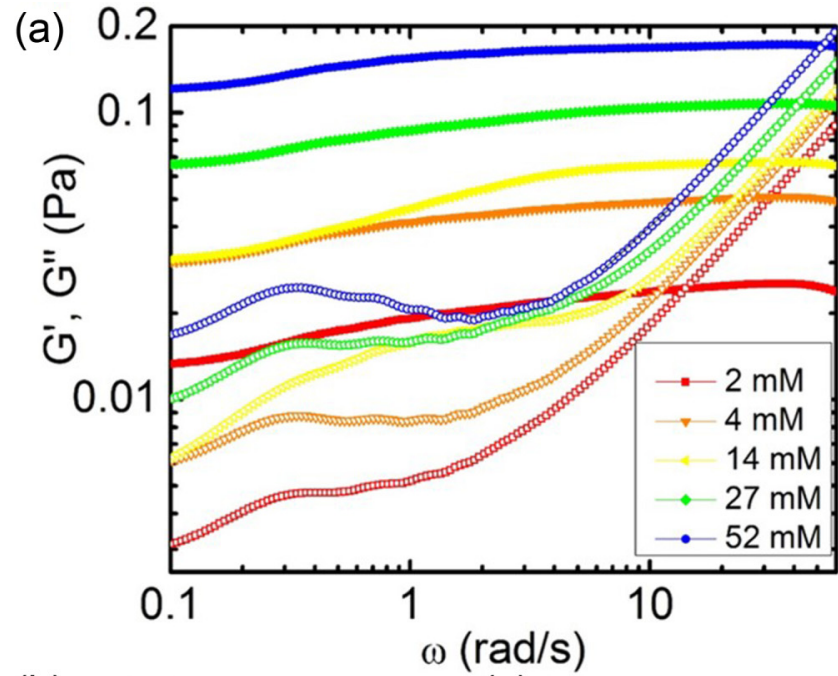

(b)
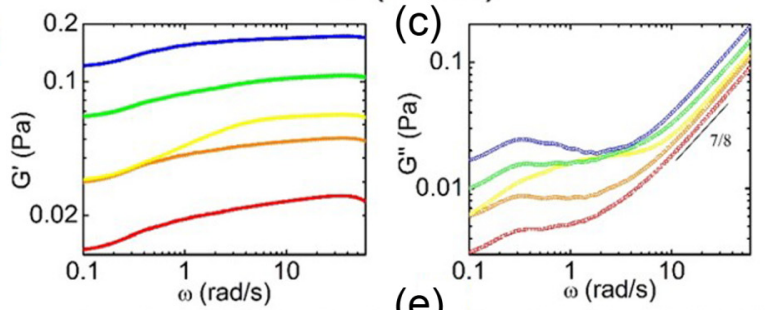

(d)
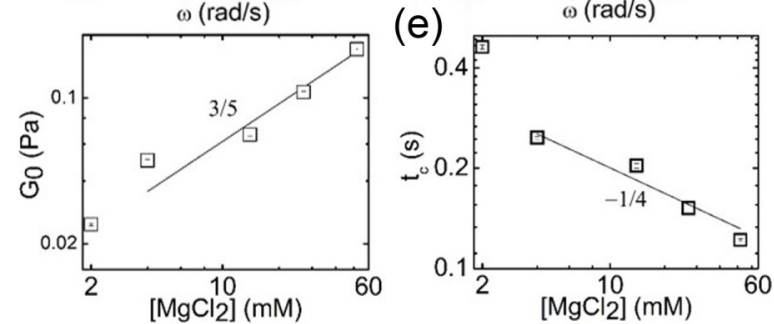

FIG. 2. Linear frequency-dependent viscoelastic moduli of actin networks exhibit strong dependence on $\mathrm{Mg}^{2+}$ concentration indicative of counterion crosslinking. (a) Frequency-dependent storage and loss moduli $\left[G^{\prime}(\omega), G^{\prime \prime}(\omega)\right]$ for actin networks in the presence of varying $\mathrm{MgCl}_{2}$ concentrations as indicated in the legend. (b) All $G^{\prime}(\omega)$ curves exhibit elastic plateaus at high frequencies with magnitudes that increase with increasing $\left[\mathrm{MgCl}_{2}\right]$. (c) $G^{\prime \prime}(\omega)$ curves exhibit local maxima at low frequencies and a power-law rise at high frequencies with the exponent listed. (d) Plateau modulus $G_{0}$ vs $\left[\mathrm{MgCl}_{2}\right]$ exhibits a power-law dependence with $\sim 3 / 5$ exponent as shown. (e) The crossover time $t_{c}=2 \pi / \omega_{c}$ at which the magnitude of $G^{\prime \prime}$ exceeds that of $G^{\prime}$ [as shown in (a)] displays a power-law dependence on $\left[\mathrm{MgCl}_{2}\right]$ with $-1 / 4$ exponent for $\left[\mathrm{MgCl}_{2}\right]>2 \mathrm{mM}$.

series or $z$-stack, averaged over frames or $z$, respectively, was computed to produce the $512 \times 512$ projection images [Fig. 1(b)].

\section{RESULTS AND DISCUSSION}

To characterize the impact of counterion concentration on the mechanical response of actin networks, we first evaluate the frequency-dependent linear viscoelastic moduli, $G^{\prime}(\omega)$ and $G^{\prime \prime}(\omega)$. As shown in Fig. 2, $G^{\prime}(\omega)$ and $G^{\prime \prime}(\omega)$ for all networks display a similar frequency dependence: $G^{\prime}$ exhibits a slow rise at low frequencies followed by a frequencyindependent elastic plateau $G_{0}$ [Fig. 2(b)] while $G^{\prime \prime}$ initially 
scales as $\sim \omega^{2 / 5}$ at low frequencies, followed by a local maximum, after which it scales as $\sim \omega^{7 / 8}\left[\right.$ Fig. 2(c)]. Further, $G^{\prime}>$ $G^{\prime \prime}$ for most of the frequency regime, but all curves exhibit a crossover to $G^{\prime \prime}>G^{\prime}$ at high frequencies. These general features are markedly similar to those reported for actin networks crosslinked by ABPs such as $\alpha$-actinin [29], indicating that bundle connectivity via crosslinking plays an important role in the mechanical response. While the frequency dependence displayed in Fig. 2 is similar for all networks, the magnitudes of $G^{\prime}$ and $G^{\prime \prime}$, as well as the time $\tau_{c}=2 \pi / \omega_{c}$ at which the crossover to $G^{\prime \prime}>G^{\prime}$ occurs, depends strongly on $\left[\mathrm{Mg}^{2+}\right]$.

The magnitude of $G_{0}$ increases as $\left[\mathrm{Mg}^{2+}\right]$ increases from 2 to $52 \mathrm{mM}$, following a power-law relation $G_{0} \sim\left[\mathrm{Mg}^{2+}\right]^{3 / 5}$. At the lowest salt concentration $(2 \mathrm{mM})$ we expect minimal bundling and crosslinking as this is the standard concentration used when realizing entangled solutions of single actin filaments. Indeed, we find that $G_{0}$ for $2 \mathrm{mM} \mathrm{Mg}^{2+}$ is comparable to the theoretically predicted value $G_{0} \approx k T / \xi^{14 / 5} l_{p}^{1 / 5} \approx$ $0.01 \mathrm{~Pa}$ for a 5.8- $\mu \mathrm{M}$ entangled actin solution [30]. However, as $\mathrm{Mg}^{2+}$ increases we expect crosslinking and bundling via counterion crossbridges to occur $[7,8]$. Thus, to estimate the expected dependence of $G_{0}$ on $\left[\mathrm{Mg}^{2+}\right]$, we turn to the predicted scaling of $G_{0}$ with persistence length $l_{p}$ for a crosslinked actin network $G_{0} \sim l_{p}^{2}$ [24]. If we consider the previously reported persistence lengths for salt-induced actin bundles $\left(\sim 4 \times l_{p}\right.$ of a single actin filament), then we expect $G_{0}$ to increase $\sim 16$-fold from the value for an entangled actin solution. This predicted value of $\sim 0.16 \mathrm{~Pa}$ is quite close to our measured value $G_{0} \approx 0.17 \mathrm{~Pa}$ for $52 \mathrm{mM} \mathrm{Mg}^{2+}$.

We also evaluate the crossover time $t_{c}=2 \pi / \omega_{c}$, which indicates the timescale at which the dynamics of single filaments or bundles, rather than the connected network, dictates the mechanics [31]. For entangled polymers $\tau_{c}$ is a measure of the predicted entanglement time $\tau_{e}$ while for crosslinked networks $t_{c}$ is related to the relaxation rate of single fibers in the network [10]. For the lowest $\mathrm{Mg}^{2+}$ concentration, we measure $t_{c} \approx$ $0.46 \mathrm{~s}$, which is comparable to the predicted entanglement time for our system $\tau_{e} \approx \beta \zeta \xi^{16 / 5} l_{p}^{1 / 5} \approx 0.25 \mathrm{~s}$, where $\zeta$ is the friction coefficient. The crossover time decreases with increasing $\mathrm{Mg}^{2+}$ and for $\left[\mathrm{Mg}^{2+}\right]>2 \mathrm{mM}$ follows the scaling $t_{c} \sim\left[\mathrm{Mg}^{2+}\right]^{-1 / 4}$. As previously predicted [5], as bundles become stiffer and more connected they exhibit extended elastic regimes, pushing the high-frequency scaling regime to higher frequencies, i.e., lower $t_{c}$ values.

Together our linear response results suggest that both increased connectivity (crosslinking) and increased persistence length (bundling) of actin fibers enables the strongly elastic response displayed for high salt networks in the linear regime. To determine the extent to which this salt-induced elasticity exhibited in the linear regime is robust to nonlinear forcing, we turn to our nonlinear microrheology results.

We previously found that nonlinear strains were sufficient to break biotin-NeutrAvidin crosslinks in actin networks, leading to stress yielding, plasticity, and stress dissipation [32]. In contrast, as shown in Fig. 3, we find that both the magnitude and slope of the induced force increase substantially with increasing $\left[\mathrm{Mg}^{2+}\right]$ with no signs of dissipation. Only the lowest salt conditions $(\leqslant 4 \mathrm{mM})$ exhibit yielding to a primarily viscous (strain-independent) plateau regime, whereas for $\left[\mathrm{Mg}^{2+}\right] \geqslant 14 \mathrm{mM}$ the force continues to increase

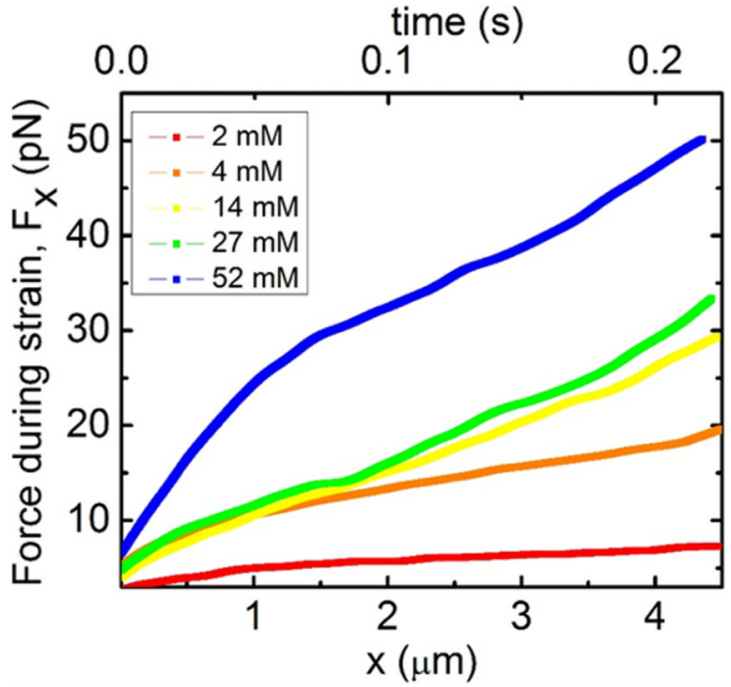

(b)
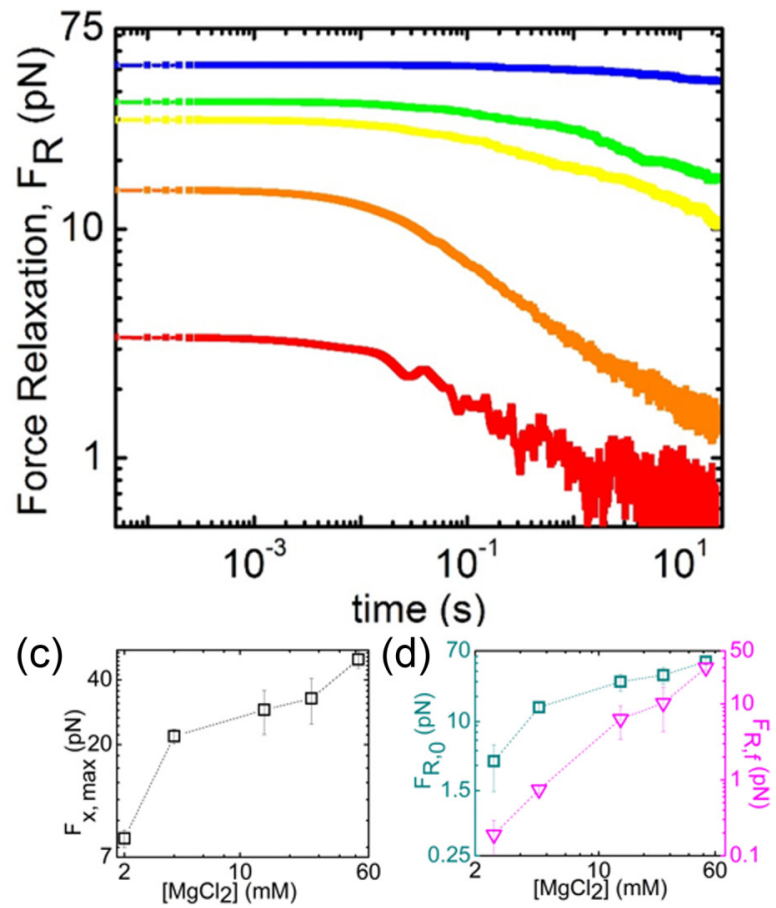

FIG. 3. Nonlinear force response of actin networks displays a marked increase in elasticity with increasing counterion concentration. (a) Force $F_{x}$ exerted by actin networks in the presence of varying $\mathrm{MgCl}_{2}$ concentrations (listed in the legend) to resist a probe dragged through the network over a distance $x$. For $\left[\mathrm{MgCl}_{2}\right]>4 \mathrm{mM}$, curves exhibit a near elastic response (i.e., linear dependence on $x$ ). (b) Corresponding relaxation of induced force $F_{R}$ following strain shows minimal dissipation for $\left[\mathrm{MgCl}_{2}\right]>4 \mathrm{mM}$. (c) The maximum force $F_{\max }$ attained during the strain [curves displayed (a)] vs $\left[\mathrm{MgCl}_{2}\right]$. (d) The relaxation force at the beginning $\left(F_{R, 0}\right.$, lefthand axis, cyan squares) and end $\left(F_{R, f}\right.$, right-hand axis, magenta triangles) of the relaxation phase as a function of $\left[\mathrm{MgCl}_{2}\right]$. As shown, the resistive force and the degree of force dissipation are strongly dependent on the density of counterions.

nearly linearly, reaching values $\sim 7$-fold larger than for low salt conditions [Fig. 3(c)]. Similarly, following the strain, the force the network exerts on the bead dissipates nearly completely for low salt conditions as the network relaxes back 

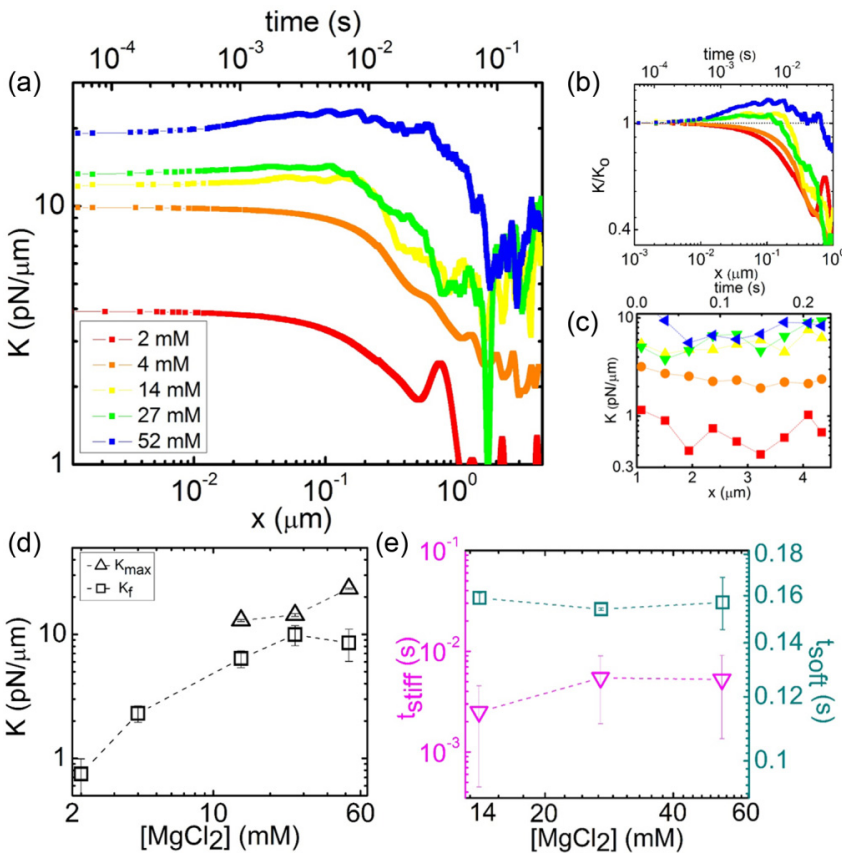

FIG. 4. Counterion bundling enables stress-stiffening and sustained elasticity above a critical $\mathrm{Mg}^{2+}$ concentration. (a) The differential modulus $K=d F_{x} / d x$ of the force curves shown in Fig. 3 as a function of strain distance $x$ for varying $\left[\mathrm{MgCl}_{2}\right]$ displayed in the legend. The inset shows the $K$ value at the beginning of the strain $K_{0}$ vs $\left[\mathrm{MgCl}_{2}\right]$. (b) $K / K_{0}$ displayed for the first $1 \mu \mathrm{m}$ of strain. The horizontal dashed line at $K / K_{0}=1$ guides the eye to show stress-stiffening $\left(K / K_{0}>1\right)$ vs stress-softening $\left(K / K_{0}<1\right)$ regimes. As shown, networks display stress-stiffening above a critical $\mathrm{MgCl}_{2}$ concentration $c_{b}$. (c) $K$ vs $x$ for the last $4 \mu \mathrm{m}$ of the strain, with points displaying $K$ values averaged over every $0.5 \mu \mathrm{m}$ to reduce noise. Above $c_{b}$, networks exhibit sustained elasticity for the duration of the strain. (d) The maximum $K$ value reached for stress-stiffening networks $\left(K_{\max }\right.$, open triangles) and final value at the end of the strain $\left(K_{f}\right.$, solid squares) vs $\left[\mathrm{MgCl}_{2}\right]$. (e) The timescales at which $K_{\max }$ is reached ( $t_{\text {stiff }}$, magenta triangles) and $K$ reaches the terminal strain-independent regime seen in (c) $\left(t_{\text {soft }}\right.$, cyan squares).

to steady state, whereas the highest salt concentration exhibits nearly complete elastic mechanomemory with the final force reached at the end of the 30-s relaxation phase dropping by less than $2 \%$ from the initial value [Fig. 3(b)]. Notably, the elasticity and strength that emerges with increased salt concentration is markedly stronger than that previously reported for actin networks crosslinked and/or bundled by ABPs [32].

To further quantify the nonlinear $\left[\mathrm{Mg}^{2+}\right]$-dependent stiffness, we evaluate the effective differential modulus $K=$ $d F / d x$. As shown in Fig. 4 , the initial stiffness $K_{0}$ steadily increases as $\left[\mathrm{Mg}^{2+}\right]$ increases up to a factor of $\sim 5$, similar to the dependence of $G_{0}$. Shortly after this initial point, $K$ separates into two distinct classes of behavior: stressstiffening and sustained elasticity for $\left[\mathrm{Mg}^{2+}\right] \geqslant 14 \mathrm{mM}$ versus stress-softening and yielding for $\left[\mathrm{Mg}^{2+}\right] \leqslant 4 \mathrm{mM}$. Stiffening $(d F / d x>0)$ and softening $(d F / d x<0)$, best shown in Fig. 4(b), is typically attributed to filament stretching and bending, respectively. Both bundling, which stiffens actin fibers, and crosslinking, which suppresses lateral motion, would prohibit bending modes. Likewise, sustaining an elastic response over the entire strain rather than yielding to a mostly viscous regime [Figs. 4(c) and 4(d)] suggests that the filaments cannot disentangle and rearrange themselves to alleviate stress. Crosslinking and bundling would prevent this source of dissipation seen in entangled actin networks [33]. As such, our data suggest that the critical $\mathrm{Mg}^{2+}$ concentration for the onset of substantial bundling $c_{b}$ is on the order of $\sim 10 \mathrm{mM}$, at the lower end of the broad range of previously reported values of $10-25 \mathrm{mM}$ [2,7-9], demonstrating that concentrations well below the upper bound of this range are sufficient.

We also evaluate the magnitude of $K$ when it reaches peak stiffness $K_{\max }$ and terminal stiffness $K_{t}$ [Figs. 4(c) and 4(d)], as well as the time at which each of these occurs, $t_{\text {stiff }}$ and $t_{\text {soft }}$, respectively. Above the critical bundling concentration, these parameters are surprisingly unaffected by changes in $\left[\mathrm{Mg}^{2+}\right]$, suggesting that bundling may contribute an "all-or-nothing" effect to nonlinear mechanics-either suppressing bending and dissipation or not. Previous studies on the size and stiffness of $\mathrm{Mg}^{2+}$-induced bundles have reported similar insensitivities or nonmonotonic dependences on salt concentration $[7-9,16]$. One explanation for this insensitivity, as described in Ref. [16], is that beyond a critical bundle thickness, further increases in salt concentration instead promote the formation of junctions between distinct bundles as well as branching of bundles into separate connected bundles. The net result is that as salt increases, the density of connections between bundles continuously increases (via junctions and branching) while the bundle thickness remains fixed.

The timescales $t_{\text {stiff }}$ and $t_{\text {soft }}$ can shed further light on the mechanisms driving the nonlinear mechanics exhibited. The average measured values are $t_{\text {stiff }} \approx 4 \mathrm{~ms}$ and $t_{\text {soft }} \approx 0.2 \mathrm{~s}$. We previously showed that highly entangled actin networks stress-stiffen over a time comparable to the fastest predicted relaxation time for actin networks, the mesh time $\tau_{\text {mesh }}$, whereas softening occurs over a time comparable to the entanglement time $\tau_{e}$ [30]. For entangled actin at $5.8 \mu \mathrm{M}, \tau_{\text {mesh }} \approx$ $\beta \zeta \xi^{4} l_{p}^{-1} \approx 0.026 \mathrm{~s}$ and $\tau_{e} \approx 0.25 \mathrm{~s}$. However, as above, if we consider that bundles increase $l_{p}$ by $\sim 4 \times$, then we can estimate $\tau_{\text {mesh }} \approx 6.5 \mathrm{~ms}$ and $\tau_{e} \approx 0.19 \mathrm{~s}$, which are remarkably close to our measured timescales $t_{\text {stiff }}$ and $t_{\text {soft }}$. These collective results indicate that in the nonlinear regime networks above $c_{b}$ behave as entangled networks of stiff fibers with no signatures of crosslinking. Thus, bundle formation, which stiffens network fibers, rather than crosslinking that connects fibers, plays the dominate role in the increased stiffness and elasticity in response to nonlinear forcing. Conversely, as described above, the linear response (Fig. 2) appears to be dictated primarily by crosslinking with bundling playing a secondary role.

We next turn to the force relaxation $F_{R}$ following strain to determine the extent to which the network can alleviate stress over time (Fig. 5). For networks below $c_{b}, F_{R}$ follows a power-law decay with an exponent of $\sim 0.3$, quite close to previously reported nonlinear microrheology results for entangled actin [25]. However, above $c_{b}$ networks exhibit very little relaxation with $F_{R}$ dropping by less than a factor of 2 (i.e., $F_{R, f} / F_{R, 0}>0.5$ ) over the entire 30 -s relaxation phase [Fig. 5(b)]. In fact, for the highest $\left[\mathrm{Mg}^{2+}\right]$ the force drops by only $2 \%$, compared to a $\sim 10$-fold drop for networks below 


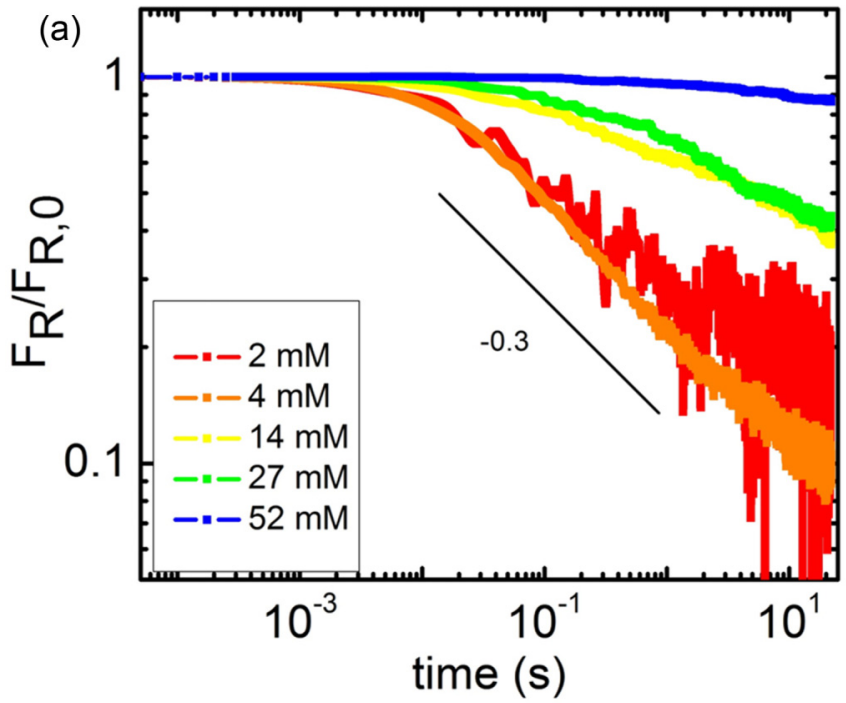

(b)
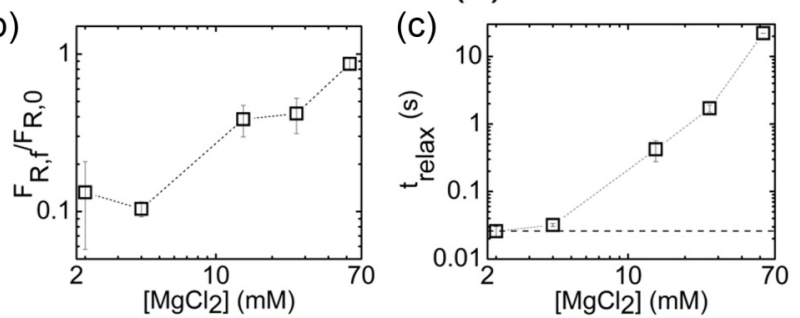

FIG. 5. Force relaxation of actin networks following nonlinear strain is suppressed by counterion-induced bundling. (a) Force relaxation $F_{R}$ shown in Fig. 3(b), normalized by the force immediately following the strain $F_{R, 0}$ for $\mathrm{MgCl}_{2}$ concentrations listed in the legend. The black line represents power-law scaling with the exponent listed. (b) The ratio of the force reached at the end the relaxation phase $F_{R, f}$ to the initial force $F_{R, 0}$ as a function of $\left[\mathrm{MgCl}_{2}\right] . F_{R, f} / F_{R, 0}=1$ for a completely elastic system while $F_{R, f} / F_{R, 0}=0$ for a system with complete dissipation. (c) The relaxation timescale $t_{\text {relax }}$, defined as the time required for the force to decay to $68.3 \%$ of the initial value $F_{0}$, increases over two orders of magnitude as $\left[\mathrm{MgCl}_{2}\right]$ exceeds the critical bundling concentration $c_{b}$. The horizontal dashed line indicates the predicted mesh time $\tau_{\text {mesh }} \approx 0.026 \mathrm{~s}$.

$c_{b}$. This extreme mechanomemory and elasticity is much stronger than reported in previous studies on crosslinked actin networks, which all show plasticity and dissipation [32,34].

We also compute the time for networks to start alleviating stress, which we quantify as the time $t_{\text {relax }}$ at which the force drops to $68.3 \%$ of its value immediately following strain $\left(F_{R, 0}\right)$. As shown, $t_{\text {relax }}$ for networks below $c_{b}$ is quite close to the predicted mesh time $\tau_{\text {mesh }} \approx 0.026 \mathrm{~s}$, suggesting that below $c_{b}$ neither bundling nor crosslinking is pervasive or strong enough to contribute to the nonlinear response. However, as $\left[\mathrm{Mg}^{2+}\right]$ becomes larger than $c_{b}$, $t_{\text {relax }}$ increases by two to three orders of magnitude. These timescales are on the order of previously reported values of $\sim 10 \mathrm{~s}$ for the disengagement time (i.e., the time for an entangled filament to reptate out of its confining tube) for entangled actin [35]. This agreement suggests that the very limited dissipation that does occur in bundled networks following nonlinear forcing is likely via reptation of bundled fibers. As such, it appears that it is bundling rather than crosslinking that is so effective

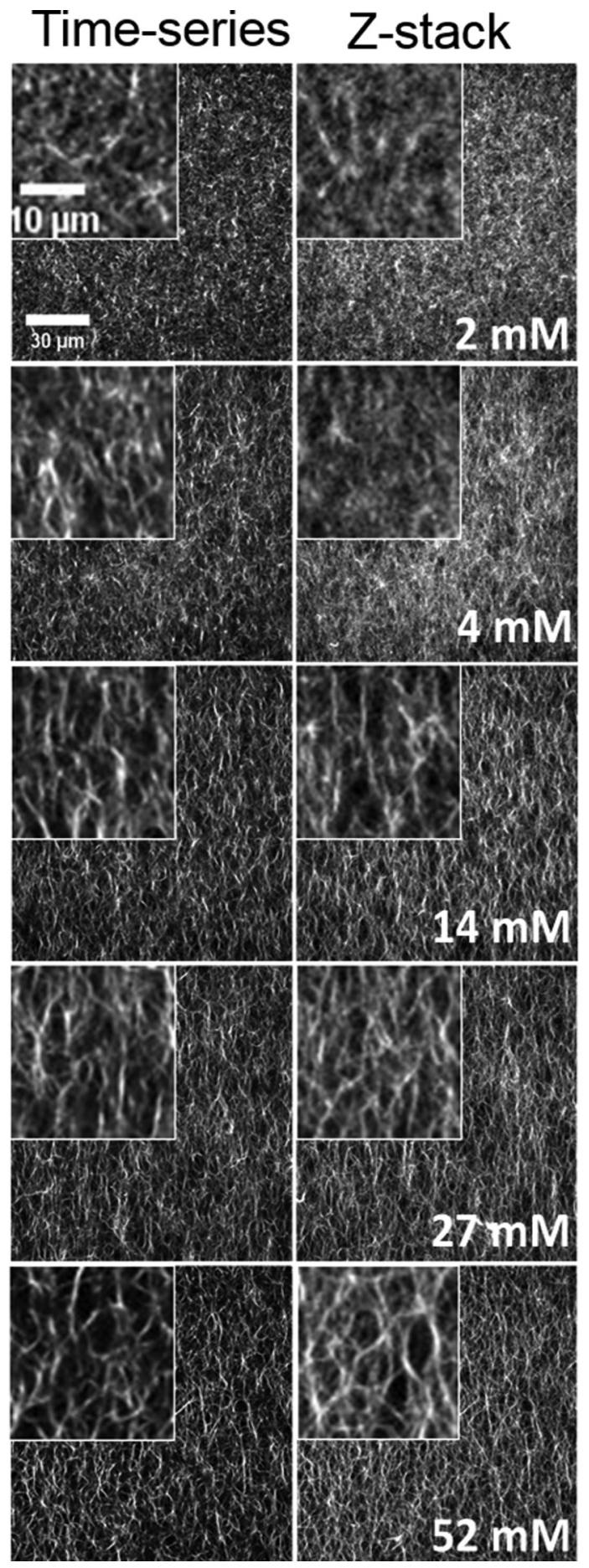

FIG. 6. Increasing concentrations of $\mathrm{Mg}^{2+}$ induce crosslinking and bundling in actin networks. Images shown are mean intensity projections of time-series (left) and $z$-stacks (right) of fluorescentlabeled actin networks acquired using a Nikon A1R laser scanning confocal microscope with $60 \times 1.4 \mathrm{NA}$ objective and QImaging CCD camera. Each row corresponds to a different $\mathrm{MgCl}_{2}$ concentration as listed in the corresponding right panel. Each time-series image is a projection of 900 images collected at $15 \mathrm{fps}$ for $60 \mathrm{~s}$ while each $z$-stack is a projection of 100 images separated in $z$ by $0.2 \mu \mathrm{m}$. The insets are $3 \times$ zoom-ins of each network. Time-series projections show a continuous decrease in network mobility while $z$-stacks show a discrete shift from networks of single filaments to bundled networks for concentrations above $4 \mathrm{mM} \mathrm{MgCl}_{2}$. 

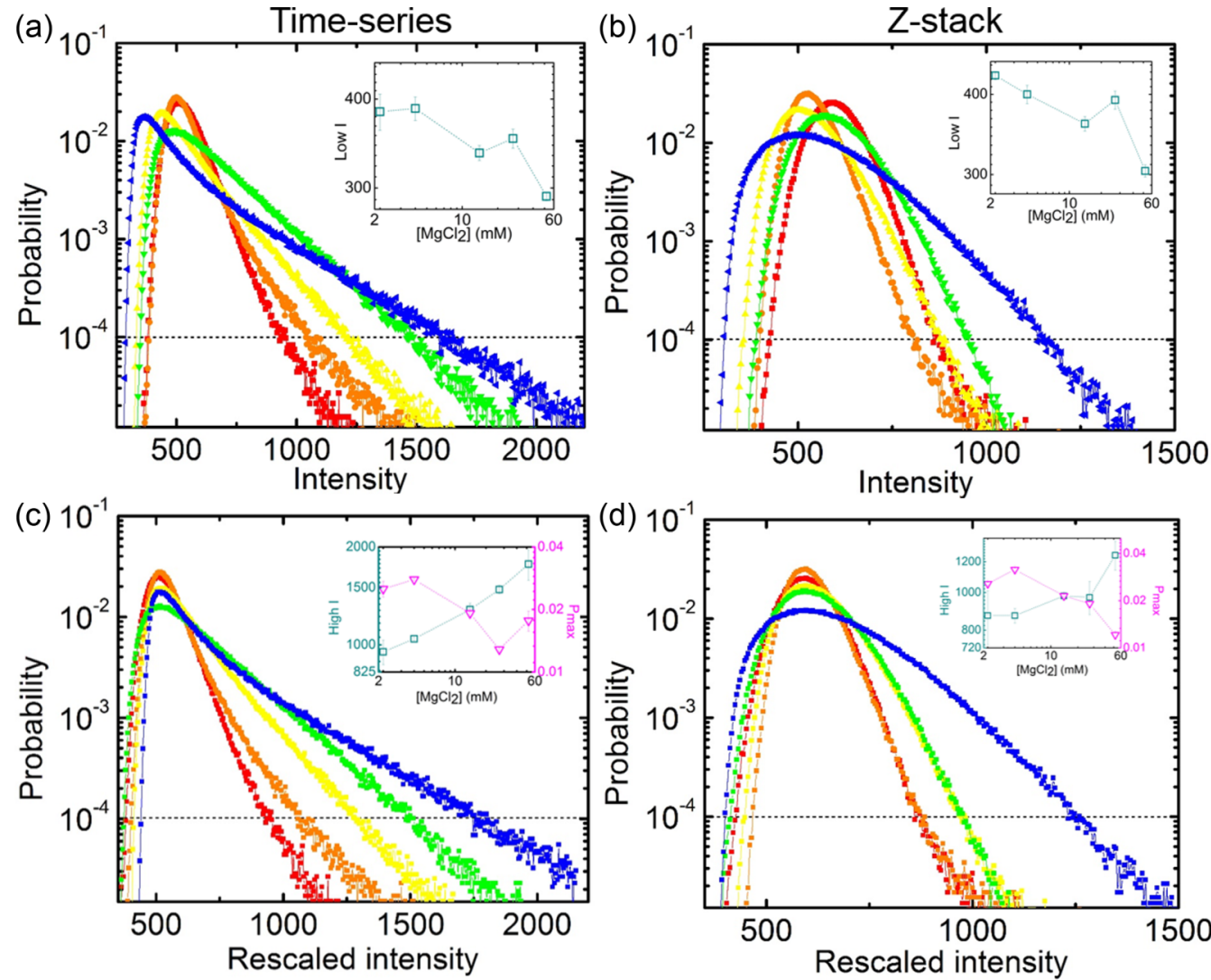

FIG. 7. Probability distributions of pixel intensities show increased rigidity and bundling of actin networks as $\left[\mathrm{Mg}^{2+}\right]$ increases. (a), (b) Probability distributions of pixel intensities from projection images of (a) time-series and (b) z-stacks shown in Fig. 6. Color coding is as in Figs. 2-5. The dashed horizontal line denotes the threshold probability value at which we evaluate the low- and high-intensity values for each distribution (labeled Low I and High I in the insets). Insets: Threshold low intensity vs $\left[\mathrm{MgCl}_{2}\right]$ determined from the corresponding distribution. (c), (d) Distributions from (a) and (b) rescaled such that their peaks align. Insets: Threshold high intensity (High I, cyan squares) and maximum probability $\left(P_{\max }\right.$, magenta triangles) vs $\left[\mathrm{MgCl}_{2}\right]$ as determined from the corresponding distribution.

at suppressing nonlinear relaxation modes and thus conferring mechanomemory.

To connect the intriguing mechanics described above with the network structure and mobility we collect time-series and three-dimensional image stacks ( $z$-stacks) of fluorescentlabeled networks using laser scanning confocal microscopy as described in Sec. II C. In the time-series projections shown in Fig. 6, networks with more Brownian fluctuations of mobile filaments should exhibit more gray pixels (lower contrast) and appear more uniform (lower pixel-to-pixel variation), whereas rigid networks should show more black and white (higher contrast) and structure (larger pixel-to-pixel variation). Similarly, $z$-stack projections for networks of bundled filaments manifest as more black and white pixels and structure, whereas networks of single filaments have lower contrast and more uniformity. As shown, for concentrations below $c_{b}$, networks appear to be comprised almost entirely of single "floppy" filaments that have ample Brownian fluctuations whereas networks above $c_{b}$ appear to be more rigid and comprised largely of bundles of filaments.

However, upon closer inspection there is a notable difference in the concentration dependence for projections of time-series versus $z$-stacks. Time-series projections show a steady increase in contrast and structure as $\left[\mathrm{Mg}^{2+}\right]$ increases, similar to our linear regime results (i.e., $G_{0}, t_{\mathrm{c}}$ ) that display a power-law dependence on $\left[\mathrm{Mg}^{2+}\right]$ (Fig. 2). On the other hand, $z$-stack projections exhibit an "all-or-nothing" effect similar to our nonlinear microrheology results, i.e., $K_{\max }, K_{t}$, $t_{\text {relax }}, F_{R, f} / F_{R, 0}$ (Figs. 3 and 4 ). Namely, contrast and pixelto-pixel variation do not appear to increase proportionally with $\left[\mathrm{Mg}^{2+}\right]$, rather there is a notable structural shift between networks above and below $c_{b}$, but little variation in contrast and structure is seen between networks that are either above or below $c_{b}$. Recall that time-series are sensitive to mechanisms that suppress temporal fluctuations such as crosslinking of filaments or bundles, whereas $z$-stacks are only sensitive to structural changes such as bundling. As such, our imaging results directly support our argument that the linear rheology is most sensitive to crosslinking density, which continuously increases with increasing $\left[\mathrm{Mg}^{2+}\right]$. Conversely, the nonlinear mechanics are dictated largely by the increased stiffness of bundles which appears to saturate soon after reaching the critical concentration-providing the all-or-nothing effect seen.

Finally, it is important to point out that given the dramatic effect of $\left[\mathrm{Mg}^{2+}\right]$ on the linear and nonlinear mechanics, the variations in images are surprisingly small. Typically, large changes to rheological properties are accompanied by mesoscale changes to the network structure whereas here 
modest microscale bundling and crosslinking appear to be sufficient.

To further quantify the $\mathrm{Mg}^{2+}$-dependent structural changes seen in Fig. 6 and corroborate our argument above, we evaluate the probability distribution of intensity values for the time-series and $z$-stack projections (Fig. 7). As shown, for $\left[\mathrm{Mg}^{2+}\right]>c_{b}$ the intensity distributions for both time-series and $z$-stack projections are broader than for networks below $c_{b}$, with higher low-intensity values and larger high-intensity tails. These features demonstrate that high- $\mathrm{Mg}^{2+}$ conditions $\left(\left[\mathrm{Mg}^{2+}\right]>c_{b}\right)$ indeed produce multifilament bundles that are brighter and less mobile than single filaments (high-intensity tails) and the apparent voids in the network are more static and empty (higher low-intensity probabilities). We quantify these features by determining the intensity values for each distribution at a threshold probability value of $10^{-4}(\sim 1 \%$ of the peak probability $P_{\max }$, shown in insets of Fig. 7). Figures 7(a) and 7(b) show that for $52 \mathrm{mM} \mathrm{Mg}^{2+}$ the low-intensity threshold values ("Low I") are $32 \%$ and $29 \%$ lower than for $2 \mathrm{mM} \mathrm{Mg}^{2+}$ in time-series and $z$-stack projections, respectively. Likewise, the high-intensity threshold values ("High I") are $46 \%$ and $30 \%$ higher for $52 \mathrm{mM} \mathrm{Mg}^{2+}$ compared to $2 \mathrm{mM}$.

The broader the distribution (more dark and light pixels), the lower the peak probability value must be, so evaluating $P_{\max }$ quantifies this counterion-dependent breadth. To best visualize variations in $P_{\max }$, as well as the width of the distributions, we rescale the distributions such that their peaks align [Figs. 7(c) and 7(d)]. Upon rescaling we see that indeed the peak heights decrease as the counterion concentration increases, with $P_{\max }$ values that are $30 \%$ and $53 \%$ lower for $52 \mathrm{mM} \mathrm{Mg}^{2+}$ than for $2 \mathrm{mM} \mathrm{Mg}^{2+}$ in time-series and $z$-stack projections, respectively.

However, the more dramatic feature is the variation in highintensity tails in time-series compared to $z$-stacks. For timeseries projections the high-intensity tails steadily increase with increasing $\left[\mathrm{Mg}^{2+}\right]$ while for $z$-stack projections the tails for networks below $c_{b}(2$ and $4 \mathrm{mM})$ collapse to a single value, as do two of the three networks above $c_{b}(14$ and $27 \mathrm{mM})$. This effect is in line with what we would expect for a time-series compared to $z$-stacks if the density of crosslinking increased steadily with $\left[\mathrm{Mg}^{2+}\right]$ while the transition to bundling was discrete. As described above and suggested in Ref. [16], one mechanism that could allow for such an effect is the increasing density of junctions and branching between growing bundles. Namely, as $\left[\mathrm{Mg}^{2+}\right]$ increases, bundles initially grow thicker until they reach a finite width, presumably occurring at $c_{b}$. At the same time the frequency of bundle-bundle junctions and bundle branching events, both of which are effectively crosslinking events, increases. However, after reaching $c_{b}$, a further increase in $\left[\mathrm{Mg}^{2+}\right]$ only serves to increase the density of junctions and branching nodes. The net effect on mechanics is a continuously increasing contribution from crosslinking yet a discrete all-or-nothing contribution from bundling as $\left[\mathrm{Mg}^{2+}\right]$ increases.

\section{CONCLUSIONS}

Actin bundling and crosslinking provides cells with tensile strength and mobility and plays critical roles in diverse mechanical processes such as mechanotransduction and division. One simple mechanism cells utilize to bundle and crosslink actin is to use divalent counterions to form crossbridges between actin filaments. Counterion crossbridges can also be exploited in materials engineering to tune the stiffness and connectivity of biopolymer and polyelectrolyte networks. However, the impact of counterion crossbridges on the mechanics of actin networks had yet to be investigated.

Here, we have addressed this need by using optical tweezers microrheology to characterize the linear and nonlinear mechanical response of entangled actin networks in the presence of varying concentrations of $\mathrm{Mg}^{2+}$. We have shown that above a critical counterion concentration $c_{b}$, bundle formation enables networks to exhibit stress-stiffening and robust elasticity that can sustain nonlinear forcing. Unlike the discrete shift seen in the nonlinear response, in the linear regime the apparent stiffness and elasticity of the network increases steadily with increasing $\left[\mathrm{Mg}^{2+}\right]$, owing to the counterionenabled crosslinking of actin filaments and bundles. Notably, these mechanical changes are significantly stronger than those previously reported for actin crosslinked or bundled via ABPs.

To corroborate these results and connect mechanics to structure, we performed laser scanning confocal microscopy experiments to characterize the effect of $\mathrm{Mg}^{2+}$ on the network microstructure and rigidity. Our results confirm that increasing counterion concentration serves to continuously increase the crosslinking density while inducing a discrete phase change to bundled fibers above a critical concentration. Importantly, we have also shown that networks exhibit surprisingly modest changes to the structure and mobility given the striking changes in the mechanical response that counterion crossbridges enable.

Our collective results not only provide key insights into understanding diverse cellular processes but also inform industrial applications. We further hope that our intriguing results will spark theoretical investigations to model the effect of counterion crossbridging on the mechanical response of biopolymer and polyelectrolyte networks.

\section{ACKNOWLEDGMENTS}

This research was funded by an NSF CAREER Award (No. 1255446) to R.M.R.A. and a W. M. Keck Foundation Research Grant to R.M.R.A., M.J.R., M.D., and J.L.R.
[1] R. Tellam, Biochemistry 24, 4455 (1985).

[2] S. Deshpande and T. Pfohl, PLoS One 10, e0116521 (2015).

[3] D. Strehle, J. Schnauß, C. Heussinger, J. Alvarado, M. Bathe, J. Käs, and B. Gentry, Eur. Biophys. J. 40, 93 (2011).

[4] M. Maier, K. W. Müller, C. Heussinger, S. Köhler, W. A. Wall, A. R. Bausch, and O. Lieleg, Eur. Phys. J. E 38, 50 (2015).
[5] M. Bathe, C. Heussinger, M. M. A. E. Claessens, A. R. Bausch, and E. Frey, Biophys. J. 94, 2955 (2008).

[6] J. Schnauß, T. Händler, and J. Käs, Polymers 8, 274 (2016).

[7] F. Huber, D. Strehle, and J. Käs, Soft Matter 8, 931 (2012).

[8] F. Huber, D. Strehle, J. Schnauß, and J. Käs, New J. Phys. 17, 043029 (2015). 
[9] N. Castaneda, T. Zheng, H. J. Rivera-Jacquez, H.-J. Lee, J. Hyun, A. Balaeff, Q. Huo, and H. Kang, J. Phys. Chem. B 122, 3826 (2018).

[10] Q. Wen and P. A. Janmey, Curr. Opin. Solid State Mater. Sci. 15, 177 (2011).

[11] M. M. A. E. Claessens, M. Bathe, E. Frey, and A. R. Bausch, Nat. Mater. 5, 748 (2006).

[12] F. Gittes, B. Mickey, J. Nettleton, and J. Howard, J. Cell Biol. 120, 923 (1993).

[13] F. Oosawa, Biophys. Chem. 11, 443 (1980).

[14] H. Kojima, A. Ishijima, and T. Yanagida, Proc. Natl. Acad. Sci. USA 91, 12962 (1994).

[15] J. X. Tang and P. A. Janmey, J. Biol. Chem. 271, 8556 (1996).

[16] G. H. Lai, R. Coridan, O. V. Zribi, R. Golestanian, and G. C. L. Wong, Phys. Rev. Lett. 98, 187802 (2007).

[17] M. L. Henle and P. A. Pincus, Phys. Rev. E 71, 060801(R) (2005).

[18] K. M. Schmoller, O. Lieleg, and A. R. Bausch, Biophys. J. 97, 83 (2009).

[19] O. Lieleg, J. Kayser, G. Brambilla, L. Cipelletti, and A. R. Bausch, Nat. Mater. 10, 236 (2011).

[20] J. H. Shin, L. Mahadevan, P. T. So, and P. Matsudaira, J. Mol. Biol. 337, 255 (2004).

[21] M. L. Gardel, J. H. Shin, F. C. MacKintosh, L. Mahadevan, P. Matsudaira, and D. A. Weitz, Science 304, 1301 (2004).

[22] A. F. Pegoraro, P. Janmey, and D. A. Weitz, Cold Spring Harbor Perspect. Biol. 9, a022038 (2017).
[23] N. Y. Yao, D. J. Becker, C. P. Broedersz, M. Depken, F. C. MacKintosh, M. R. Pollak, and D. A. Weitz, J. Mol. Biol. 411, 1062 (2011).

[24] C. P. Broedersz and F. C. MacKintosh, Rev. Mod. Phys. 86, 995 (2014).

[25] T. T. Falzone, S. Blair, and R. M. Robertson-Anderson, Soft Matter 11, 4418 (2015).

[26] C. D. Chapman, K. Lee, D. Henze, D. E. Smith, and R. M. Robertson-Anderson, Macromolecules 47, 1181 (2014).

[27] R. R. Brau, J. M. Ferrer, H. Lee, C. E. Castro, B. K. Tam, P. B. Tarsa, P. Matsudaira, M. C. Boyce, R. D. Kamm, and M. J. Lang, J. Opt. A: Pure Appl. Opt. 9, S103 (2007).

[28] M. Tassieri, R. M. L. Evans, R. L. Warren, N. J. Bailey, and J. M. Cooper, New J. Phys. 14, 115032 (2012).

[29] T. T. Falzone, M. Lenz, D. R. Kovar, and M. L. Gardel, Nat. Commun. 3, 861 (2012).

[30] H. Isambert and A. C. Maggs, Macromolecules 29, 1036 (1996).

[31] K. W. Müller, R. F. Bruinsma, O. Lieleg, A. R. Bausch, W. A. Wall, and A. J. Levine, Phys. Rev. Lett. 112, 238102 (2014).

[32] B. Gurmessa, S. Ricketts, and R. M. Robertson-Anderson, Biophys. J. 113, 1540 (2017).

[33] O. Lieleg, K. M. Schmoller, M. M. A. E. Claessens, and A. R. Bausch, Biophys. J. 96, 4725 (2009).

[34] S. Majumdar, L. C. Foucard, A. J. Levine, and M. L. Gardel, Soft Matter 14, 2052 (2018).

[35] B. Gurmessa, R. Fitzpatrick, T. T. Falzone, and R. M. Robertson-Anderson, Macromolecules 49, 3948 (2016). 